\title{
TCM, TTCM, BICM and BICM-ID Assisted MMSE Multi-User Detected SDMA-OFDM Using Walsh-Hadamard Spreading
}

\author{
M. Jiang, S. X. Ng, L. Hanzo ${ }^{1}$ \\ Dept. of ECS, Univ. of Southampton, SO17 1BJ, UK. \\ Tel: +44-703-593 125, Fax: +44-703-593045 \\ Email: ${ }^{1}$ lh@ecs.soton.ac.uk, http://www-mobile.ecs.soton.ac.uk
}

\begin{abstract}
Space Division Multiple Access (SDMA) aided Orthogonal Frequency Division Multiplexing (OFDM) systems assisted by efficient Multi-User Detection (MUD) techniques have recently attracted intensive research interests. Forward Error Correction (FEC) schemes and frequency-domain spreading techniques can be efficiently amalgamated with SDMA-OFDM systems for the sake of improving the achievable performance. In this contribution a Coded Modulation (CM) assisted and Minimum Mean-Square Error (MMSE) multi-user detected SDMAOFDM system combined with Walsh-Hadamard-TransformSpreading (WHTS) across a number of subcarriers is proposed. The various CM schemes used are Trellis Coded Modulation (TCM), Turbo TCM (TTCM), Bit-Interleaved Coded Modulation (BICM) and Iteratively Decoded BICM (BICM-ID), which constitute bandwidth efficient schemes that combine the functions of coding and modulation. Invoking the WHTS technique is capable of further improving the average Bit Error Rate (BER) performance of the CM-SDMA-OFDM system, since the bursty error effects imposed by the frequency-domain fading encountered are spread over the entire WHT block length, therefore increasing the chances of correcting the transmission errors by the $\mathrm{CM}$ decoders.
\end{abstract}

\section{INTRODUCTION}

Space Division Multiple Access (SDMA) based Orthogonal Frequency Division Multiplexing (OFDM) [1] communication invoking MultiUser Detection (MUD) techniques has recently attracted intensive research interests. In SDMA Multi-Input-Multi-Output (MIMO) systems the transmitted signals of $L$ simultaneous uplink mobile users - each equipped with a single transmit antenna - are received by the $P$ different receiver antennas of the Base Station (BS). At the BS the individual users' signals are separated with the aid of their unique, user-specific spatial signature constituted by their channel transfer functions or, equivalently, Channel Impulse Responses (CIRs). A variety of MUD schemes, such as the Least-Squares (LS) $[2,3]$ and Minimum Mean-Square Eтог (MMSE) [2-4] detectors, or Successive Interference Cancellation (SIC) [2-5], Parallel Interference Cancellation (PIC) $[2,5,6]$ and Maximum Likelihood Detection (MLD) $[2$, $4,7]$ schemes may be invoked for the sake of separating the different users at the BS on a per-subcarrier basis. Among these schemes, the ML detection arrangement was found to give the best performance, although this was achieved at the cost of a dramatically increased com-

Acknowledgements: The work reported in this paper has formed part of the (relevant work area) area of the (Core 2 , or 1 , or 3 , or Elective Title, as appropriate) Research Programme of the Virtual Centre of Excellence in Mobile and Personal Communications, Mobile VCE, www.mobilevce.com, whose funding support, including that of EPSRC, is gratefully acknowledged. Fully detailed technical reports on this research are available to Industrial Members of Mobile VCE. putational complexity, especially in the context of a high number of users and higher-order modulation schemes, such as 16QAM [5]. By contrast, MMSE combining exhibits the lowest complexity in this set of detectors, while suffering from a performance loss $[1,5]$.

In order to improve the achievable performance by exploiting the multi-path diversity potential offered by wideband channels, a further technique that is often used in the context of Code Division Multiple Access (CDMA) systems is constituted by the spreading of the subcarrier signals over a number of adjacent subcarriers with the aid of orthogonal spreading codes, such as Walsh-Hadamard-Transform (WHT) based codes. This technique may also be employed in multiuser SDMA-OFDM systems in the context of spreading across all or a fraction of the subcarriers [8]. Spreading across all subcarriers using a single WHT Spreading (WHTS) code is expected to result in a better averaging of the bursty error effects at the cost of a higher WHT complexity.

Furthermore, the achievable performance can be significantly improved, if Forward Error Correction (FEC) schemes, such as for example Turbo Convolutional (TC) codes [1] are incorporated into the SDMA system. Furthermore, Trellis Coded Modulation (TCM) [9, 10], Turbo TCM (TTCM) $[9,11]$, Bit-Interleaved Coded Modulation (BICM) $[9,12]$ and Iteratively Decoded BICM (BICM-ID) $[9,13]$ have attracted intensive research interests, since they are capable of achieving a substantial coding gain without bandwidth expansion.

In this contribution, we combine the above-mentioned various Coded Modulation (CM) schemes with an MMSE-MUD assisted SDMAOFDM system, in which WHT-based subcarrier spreading is used. The structure of this paper is as follows. The SDMA MIMO channel model is described in Section 2.1, while the overview of the CMassisted MMSE-SDMA-WHTS-OFDM system is given in Section 2.2, where the basic principles of CM, MMSE-based MUD and WHTbased spreading (WHTS) are also introduced. Our simulation results are provided in Section 3, while our conclusions are summarized in Section 4.

\section{SYSTEM MODEL}

\subsection{SDMA MIMO Channel Model}

Figure 1 shows an SDMA uplink MIMO channel model, where each of the $L$ simultaneous mobile users employs a single transmit antenna, while the BS's receiver exploits $P$ antennas. At the $k^{\text {th }}$ subcarrier of the $n^{\text {th }}$ OFDM symbol received by the $P$-element BS antenna array we have the complex received signal vector $\mathbf{x}[n, k]$, which is constituted by the superposition of the independently faded signals associated with the $L$ mobile users and contaminated by the Additive White Gaussian Noise (AWGN), expressed as:

$$
\mathbf{x}=\mathbf{H} \mathbf{s}+\mathbf{n},
$$




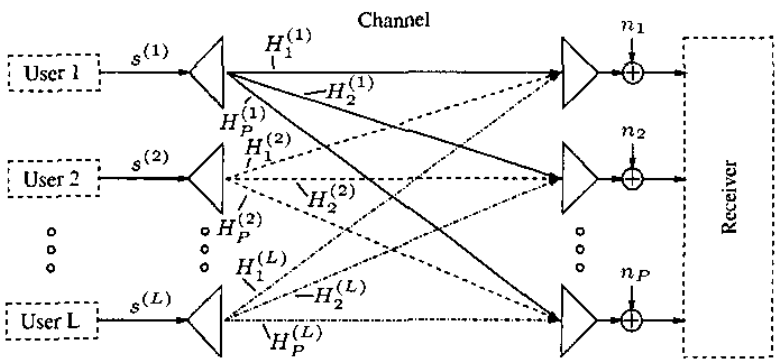

Figure 1: Schematic of the SDMA uplink MIMO channel model [1], where each of the $L$ mobile users is equipped with a single transmit antenna and the BS's receiver is assisted by a $P$-element antenna front-end.

where the $(P \times 1)$-dimensional vector $\mathbf{x}$, the $(L \times 1)$-dimensional vector $\mathbf{s}$ and the $(P \times 1)$-dimensional vector $\mathbf{n}$ are the received, transmitted and noise signals, respectively. Here we have omitted the indices $[n, k]$ for each vector for the sake of notational convenience. Specifically, the vectors $\mathbf{x}, \mathbf{s}$ and $\mathbf{n}$ are given by:

$$
\begin{aligned}
& \mathbf{x}=\left(x_{1}, x_{2}, \ldots, x_{P}\right)^{T}, \\
& \mathbf{s}=\left(s^{(1)}, s^{(2)}, \ldots, s^{(L)}\right)^{T}, \\
& \mathbf{n}=\left(n_{1}, n_{2}, \ldots, n_{P}\right)^{T} .
\end{aligned}
$$

The $(P \times L)$-dimensional matrix $\mathbf{H}$, which contains the frequencydomain channel transfer functions (FD-CHTF) of the $L$ users, is given by:

$$
\mathbf{H}=\left(\mathbf{H}^{(1)}, \mathbf{H}^{(2)}, \ldots, \mathbf{H}^{(L)}\right),
$$

where $\mathbf{H}^{(l)}(l=1 \ldots L)$ is the vector of the FD-CHTFs associated with the transmission paths from the $l^{\text {th }}$ user's transmit antenna to each element of the $P$-element receiver antenna array, which is expressed:

$$
\mathbf{H}^{(l)}=\left(H_{1}^{(l)}, H_{2}^{(l)}, \ldots, H_{P}^{(l)}\right)^{T}, \quad l=1 \ldots L .
$$

In Equations 1 to 6, we assume that the complex signal $s^{(l)}$ transmitted by the $l^{t h}$ user has zero-mean and a variance of $\sigma_{l}^{2}$. The AWGN noise signal $n_{p}$ also exhibits a zero-mean and a variance of $\sigma_{n}^{2}$. The FD-CHTFs $H_{p}^{(i)}$ of the different receivers or users are independent. stationary, complex Gaussian distributed processes with zero-mean and unit variance [8].

\subsection{CM-assisted MMSE-SDMA-WHTS-OFDM}

In Section 2.1 we have briefly reviewed the SDMA MIMO channel model, as shown in Figure 1. In Figure 2, we present the schematic of the proposed CM-assisted and MMSE multi-user detected SDMAOFDM uplink system employing WHT spreading. At the transmitter end, as seen at the top of Figure 2, the information bit sequences of the geographically separated $L$ simultaneous mobile users are forwarded to the CM [9] encoders, where they are encoded into symbols. The encoded signals $s^{(l)}(l=1 \ldots L)$ are then forwarded to the subcarrier-based WHT spreader [1], followed by the OFDM-related Inverse Fast Fourier Transform (IFFT) based modulator, which converts the frequency-domain signals to the time-domain modulated OFD symbols. The OFDM symbols are then transmitted by the Mobile Stations (MSs) to the BS over the SDMA MIMO channel. Then

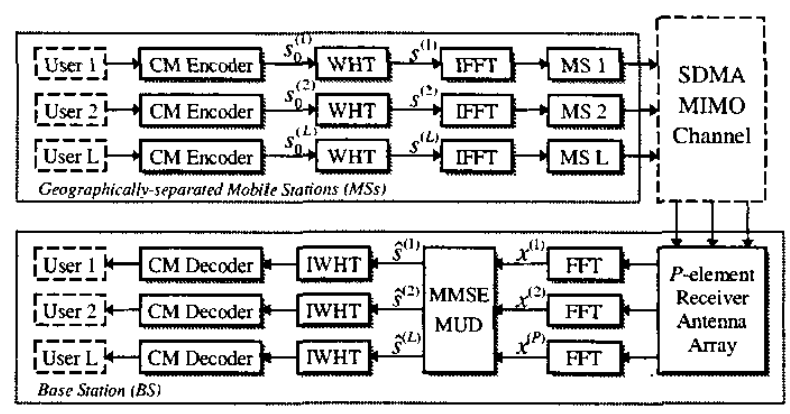

Figure 2: Schematic of the CM-assisted and MMSE multi-user detected SDMA-OFDM uplink system employing subcarrier-based WHT spreading.

each element of the receiver antenna array shown at the bottom of Figure 2 receives the superposition of the transmitted signals faded and contaminated by the channel and performs Fast Fourier Transform (FFT) based OFDM demodulation. The demodulated outputs $x^{(p)}(p=1 \ldots P)$ seen in Figure 2 are forwarded to the MMSEbased MUD [1] for separating the different users' signals. The separated signals $\hat{s}^{(l)}(l=1 \ldots L)$, namely the estimated versions of the transmitted signals, are independently despread based on the Inverse WHT (IWHT) and then decoded by the CM decoders of Figure 2. Upon employing the WHTS technique, the detrimental effects imposed on the system's average Bit Error Rate (BER) performance by the specific subcartiers which are corrupted by deep frequencydomain channel fades can be potentially improved, since the effects of the fades are spread over the entire WHT block. Hence the receiver has a better chance of recovering the impaired transmitted signals of the badly affected subcarriers, which now carry only a single chip of the WHTS code representing a subcarrier symbol.

\section{SIMULATION RESULTS}

In this section, we characterize the performance of the proposed CMassisted MMSE-SDMA-OFDM schemes in conjunction with WHTS. The channel is assumed to be OFDM symbol-invariant, implying that the taps of the impulse response are assumed to be constant for the duration of one OFDM symbol, but they are faded at the beginning of each symbol [1]. The simulation results were obtained using a 4QAM scheme communicating over the 3-path Short Wireless Asynchronous Transfer Mode (SWATM) CIR given on page 78 of [1], and the 12path COST207 [14] Hilly Terrain (HT) CIR shown on page 388 of [9], assuming that the channels' transfer functions are perfectly known. Each of the paths experiences independent Rayleigh fading having the same normalized Doppler frequencies of $f_{d}^{\prime}=1.235 \times 10^{-5}$ and $f_{d}^{\prime}=1.0 \times 10^{-5}$, for the SWATM and COST207 HT channels, respectively. The specified channel parameters are given in Table 1.

\begin{tabular}{|c|c|c|c|c|c|}
\hline Channel & $\tau_{\max }$ & $f_{d}^{\prime}$ & $n$ & $K$ & $c p$ \\
\hline SWATM & $48.9 \mathrm{~ns}$ & $1.235 \times 10^{-5}$ & 3 & 512 & 64 \\
\hline COST207 HT & $19.9 \mu \mathrm{s}$ & $1.0 \times 10^{-5}$ & 12 & 2048 & 256 \\
\hline
\end{tabular}

Table 1: Maximum path delay $\tau_{\max }$, maximum Doppler frequency $f_{d}$, normalized Doppler frequency $f_{d}^{\prime}$, number of paths $n$, FFT length $K$ and cyclic prefix length $c p$ of the SWATM [1] and COST207 HT [9] channels. 
For the various $\mathrm{CM}$ schemes used, we select the parameters so that all schemes have the same effective throughput and the same number of decoding states, hence have a similar decoding complexity. More specifically, the code memory $\nu$ is fixed to 6 for the noniterative TCM and BICM schemes, so that the number of decoding states becomes $S=2^{\nu}=64$. For the iterative TTCM and BICM-ID schemes, however, $\nu$ is fixed to 3 , while the number of iterations for these schemes is set to 4 and 8 , respectively. Hence the number of total trellis states is $2^{3} \cdot 4 \cdot 2=64$ for TTCM and $2^{3} \cdot 8 \cdot 1=64$ for BICM-ID, since there are two 8-state decoders, which are invoked in four iterations in the scenario of TTCM, while only one 8-state decoder is employed in the context of BICM-ID. Furthermore, the codeword length and channel interleaver depth for all the $\mathrm{CM}$ schemes are fixed to 1024 and 2048 symbols for communicating over the SWATM and COST207 HT channels, respectively.

\subsection{MMSE-SDMA-OFDM using WHTS}
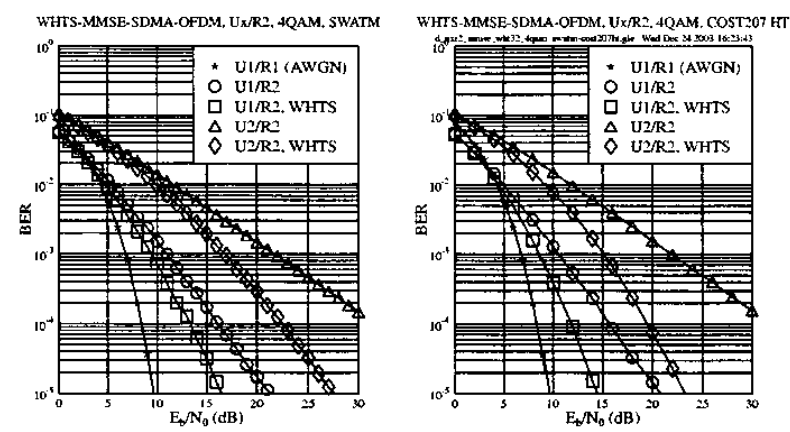

Figure 3: BER versus $E_{b} / N_{0}$ performance of the WHTS-assisted MMSE-SDMA-OFDM system employing a 4QAM scheme for transmission over the SWATM (left) and the COST207 HT (right) channels, where one or two users are supported with the aid of two receiver antenna elements. The WHT block size used is $\mathbf{3 2}$.

Figure 3 compares the BER versus $E_{b} / N_{0}$ performance of the MMSE-SDMA-OFDM system both with and without WHTS for transmission over the SWATM [1] and the COST207 HT [9] channels, respectively. As expected, the WHTS-assisted schemes perform better than their non-spread counterparts, both for one and two users. This suggests that the system's average BER performance can be improved by using WHTS, since the bursty subcarrier errors can be effectively spread across the subcarriers within the entire WHT block, which improves the CM decoder's achievable performance.

\subsection{CM-assisted MMSE-SDMA-OFDM using WHTS}

In Section 3.1 the beneficial effects of WHTS on the MMSE-SDMAOFDM system's performance have been demonstrated. Let us now combine the various CM schemes considered with the WHTS-aided MMSE-SDMA-OFDM system.

\subsubsection{Two Receiver Antenna Elements}

The BER performance of the CM-WHTS-MMSE-SDMA-OFDM system is portrayed in Figure 4. The top and bottom of the figure illustrates the corresponding results achieved in the SWATM and the
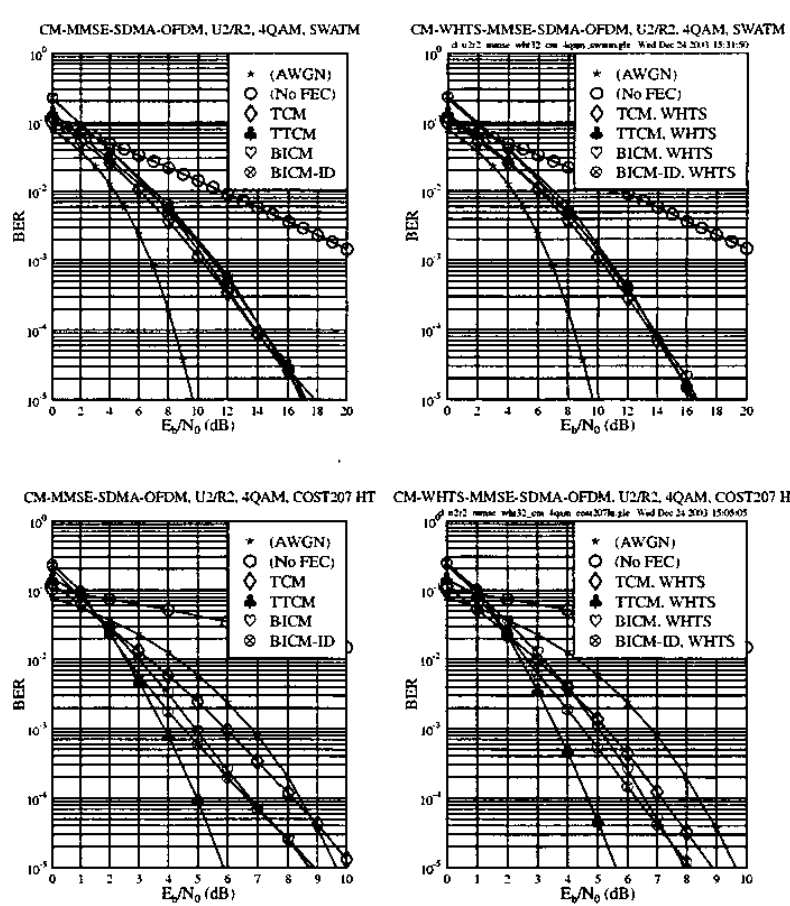

Figure 4: BER versus $E_{b} / N_{0}$ performance of the CM-assisted MMSE-SDMA-OFDM (left) and CM- and WHTS-assisted MMSE-SDMA-OFDM (right) systems employing a 4QAM scheme for transmission over the SWATM (top) and the COST207 HT (bottom) channels, where two users are supported with the aid of two receiver antenna elements. The WHT block size used is $\mathbf{3 2}$.

COST207 HT channels, respectively, while the left and right hand sides show the performance attained by the CM-MMSE-SDMA-OFDM system without and with the aid of WHTS, respectively. It transpires from the bottom of Figure 4 that the four CM-aided schemes communicating over the COST207 HT channel attain a different performance. This observation is in contrast to what was noted at the top of the Figure 4, where the performance of the various $\mathrm{CM}$-aided schemes communicating over the SWATM channel was more similar. The reason for this phenomenon is that the amplitude variation of the FDCHTFs becomes both more frequent and more dramatic, when the channel exhibits a longer path delay [9], as seen in Table 1. This characteristic will result in less burstily distributed corrupted subcarrier symbols, which hence can be more readily corrected by the channel codes, for example one of the four CM schemes. More specifically, the more prolonged error bursts imposed by the SWATM channel often overload the error correction capability of the CM schemes, regardless of which of the four CM schemes is used, since owing to the preponderance of transmission errors their trellis decoder often opts for choosing the wrong trellis path. Therefore, this phenomenon results in a similar performance for the various CM-aided schemes, when communicating over the SWATM channel. as seen in the top of Figure 4. By contrast, in the context of the COST207 HT channel such prolonged error bursts are unlikely to occur, since the faded subcarriers result in more frequent but less prolonged error bursts, which are 
reminiscent of the error distributions experienced in AWGN channels and therefore may have a higher chance of being corrected by the $\mathrm{CM}$ decoders used at the receiver. Hence, the different error-correcting capability of the various $\mathrm{CM}$ schemes becomes more explicit, as revealed at the bottom of Figure 4 .

On the other hand, it was found that the TTCM-aided scheme constitutes the best design option in terms of the BER performance, attaining an extra coding gain ranging from $2 \mathrm{~dB}$ to $4 \mathrm{~dB}$ over the other three CM-aided schemes at the BER of $10^{-5}$ without the assistance of WH'TS, while communicating over the COST207 HT channel. Furthermore, when WHTS is incorporated into the CM-MMSE-SDMAOFDM system in the context of the COST207 HT channel, as seen at the bottom of Figure 4, a further useful $E_{b} / N_{0}$ gain is achieved by most of the four schemes, especially by the TCM-aided arrangement. However, in the scenario where the SWATM channel was employed, the additional $E_{b} / N_{0}$ gain achieved by spreading in the context of the various CM- and WHTS-assisted schemes was rather modest. This result may suggest that in highly dispersive environments, such as that characterized by the 12-path COST207 HT channel, the channel-coded SDMA-OFDM system's performance may be further improved by employing WHTS. This spreading-induced $E_{b} / N_{0}$ gain was achieved, because the detrimental effects imposed on the system's average BER performance by the deeply-faded subcarriers has been spread over the entire WHT block, and these randomized or dispersed channel errors may be more readily corrected by the CM decoder.

\subsubsection{Four Receiver Antenna Elements}

Here we define the user load of an $L$-user and $P$-receiver SDMAOFDM system as:

$$
\alpha_{\mathrm{SDMAP}_{P}}=l / P, \quad l \in\{1 \ldots L\},
$$

which assumes a value of unity in case of full user load, when the number of users is equal to the number of receiver antenna elements. The left hand side of Figure 5 shows the $E_{b} / N_{0}$ crossing points of the various CM-WHTS-MMSE-SDMA-OFDM schemes at the BER of $10^{-5}$. It is shown explicitly that the performance gap between the different $\mathrm{CM}$-aided schemes increases as the user load increases. Furthermore, from Figure 5 we see that the TTCM-aided scheme performs best in high user-load scenarios, namely for $\alpha_{\mathrm{SDMA}_{4}} \geq 0.5$. In other words, the other three CM schemes, namely the TCM, BICM and BICM-ID arrangements will suffer a higher performance degradation than TTCM, when the MUD's user-separation capability erodes owing to the increased multi-user interference. Additionally, at the right hand side of Figure 5 we compare the total gain achieved by the four different $\mathrm{CM}$-aided schemes, which includes both the coding gain and the spreading-induced $E_{b} / N_{0}$ gain. As the figure indicates, the TTCM-aided scheme achieved a further $E_{b} / N_{0}$ gain of $3.76 \mathrm{~dB}, 2.38 \mathrm{~dB}$ and $2.61 \mathrm{~dB}$ over the TCM, BICM and BICM-ID aided schemes in the fully-loaded scenario, respectively. At a relatively low user load, namely for $\alpha_{\mathrm{SDMA}_{4}} \leq 0.5$, the various schemes provide a similar performance, because most of the attainable gain in the four-receiver SDMA-OFDM system has already been achieved.

As shown in Figure 5, for each of the schemes evaluated, we may notice that the performance achieved in the context of a lower user load, is better than that attained, when we have a higher user load. This phenomenon may be explained as follows. Since $P$ receiver antenna elements are invoked at the BS, there are $P$ uplink paths for each MS user having one transmit antenna. Hence the achievable space-diversity order provided by the $P$ paths remains the same for

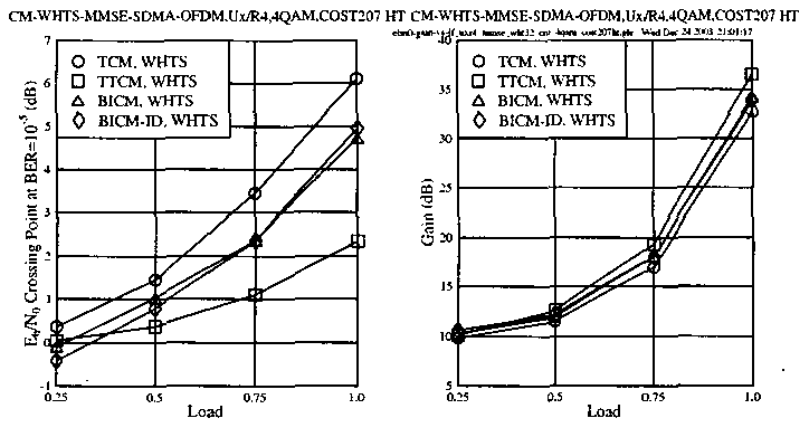

Figure 5: $\mathbf{E}_{\mathrm{b}} / \mathbf{N}_{\mathbf{0}}$ Crossing Point (left) and Gain (right) at the BER of $10^{-5}$ versus user load performance of the CM- and WHTSassisted MMSE-SDMA-OFDM system employing a 4QAM scheme for transmission over the COST207 HT channel, where one to four users are supported with the aid of four receiver antenna elements. The WHT block size used is $\mathbf{3 2}$.

each user, regardless of the total number of simultaneous users. However, when the user load is lower, i.e. the number of users supported is lower, the MMSE combiner will benefit from a higher degree of freedom in terms of the choice of the array weights optimized for differentiating the different users' transmitted signal, and thus the system becomes more efficient in terms of suppressing the detrimental fading channel effects.

\subsubsection{Effect of the WHT Block Size and Doppler Frequency}

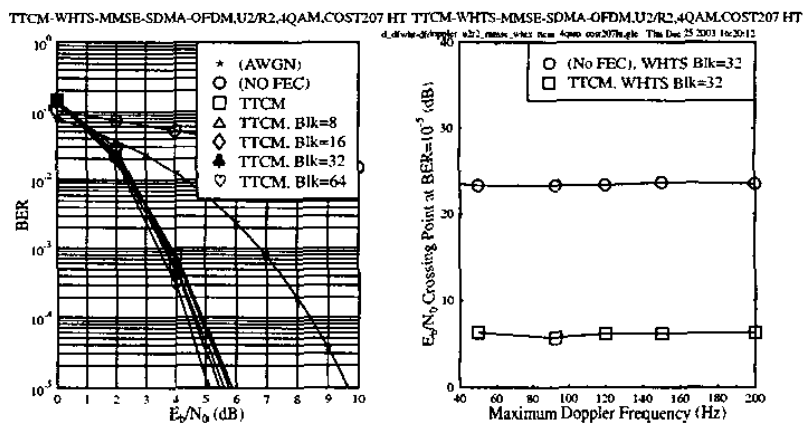

Figure 6: BER versus $\mathbf{E}_{b} / \mathbf{N}_{\mathbf{0}}$ (left) and $\mathbf{E}_{\mathbf{b}} / \mathbf{N}_{\mathbf{0}}$ Crossing Point at the BER of $10^{-5}$ versus maximum Doppler frequency (right) performance of the TTCM- and WHTS-assisted MMSE-SDMAOFDM system employing a 4QAM scheme for transmission over the COST207 HT channel, where two users are supported with the aid of two receiver antenna elements. Different WHT block sizes are used for the left hand side scenario, while for the right hand side the WHT block size used is $\mathbf{3 2}$.

In order to show the effects imposed by different-length WHTS schemes, as an example, we provide simulation results generated in the context of different WHT block sizes in the TTCM-aided scenar- 
ios, as plotted at the left hand side of Figure 6. As expected, the system's average BER performance was improved upon increasing the WHT block size, since the signals carried by the subcarriers that were gravely affected by deep channel fades were spread over a larger set of subcarriers. In this situation the frequency-domain fading of a subcarrier would obliterate a single WHT chip of a sequence conveying a modulated symbol rather than the subcarrier's symbol itself, which may be expected to mitigate the detrimental channel effects and thus assists the receiver in achieving a better performance. Furthermore, we may notice that the spreading-induced $E_{b} / N_{0}$ gains achieved by the TTCM-aided scheme when using a larger WHT block size are modest. This suggests that in the TTCM-aided SDMA-OFDM system, most of the achievable diversity gain has been attained by the time-diversity of the TTCM scheme. However, as observed at the bottom of Figure 4 where the performance of the various CM-aided schemes communicating over the COST207 HT channel were compared, the other three CM-aided schemes, for example the TCM-aided arrangement, may be capable of achieving a higher spreading-induced gain than the TTCM-assisted scheme, when invoking WHTS. Therefore, owing to their lower time-diversity and relatively more modest unspread performance, a potentially higher spreading-induced $E_{b} / N_{0}$ gain may be achieved by combining WHTS with the TCM, BICM and BICM-ID assisted schemes than in conjunction with the TTCM-aided arrangement, when a larger WHT block size is used.

In our further investigations we have generated the BER versus $E_{b} / N_{0}$ curves of the TTCM- and WHTS-assisted MMSE-SDMAOFDM system communicating over the COST207 HT channel, when the maximum Doppler frequency was varied over a range of different values. Again, the CIR of the 12-path COST207 HT channel shown on page 388 of [9] was used. At the right hand side of Figure 6 , we show the $E_{b} / N_{0}$ crossing point at $\mathrm{BER}=10^{-5}$ versus the maximum Doppler frequency for the WHTS-assisted MMSE-SDMAOFDM system both with and without the aid of TTCM, where two receivers were used for supporting two users. We conclude from the near-horizontal curves shown in the figure that the maximum Doppler frequency does not significantly affect the performance of the WHTSassisted MMSE-SDMA-OFDM system, regardless of the employment of TTCM. This is a highly desirable benefit of the error-randomizing effect of WHTS, resulting in a high robustness against the variation of the mobile speed. Moreover, as expected, the performance of the TTCM-aided scheme was consistently better, than that of the scheme using no channel coding, as evidenced by the right hand side illustration of Figure 6.

\section{CONCLUSIONS}

From the investigations conducted, we conclude that the various $\mathrm{CM}$ schemes, namely TCM, TTCM, BICM and BICM-ID are capable of substantially improving the achievable performance of SDMAOFDM systems. The employment of WHTS has the potential of further enhancing the system's performance in highly dispersive propagation environments. As a result, the TTCM- and WHTS-assisted scheme was found to be the best design option in terms of the achievable $E_{b} / N_{0}$ gain expressed in $\mathrm{dB}$, when communicating in highly dispersive environments, for example over the COST207 HT [9] channel, while carrying a high user load of $\alpha_{\mathrm{SDMA} P} \geq 0.5$.

\section{REFERENCES}

[1] L. Hanzo, M. Münster, B. Choi, and T.Keller, OFDM and MCCDMA for Broadband Multi-user Communications, WLANs and Broadcasting. IEEE Press - John Wiley \& Sons Ltd., 2003.
[2] S. Verdu, Multiuser Detection. Cambridge University Press, 1998.

[3] C. Sweatman, J. Thompson, B. Mulgrew, and P. M. Grant, "A Comparison of Detection Algorithms including BLAST for Wireless Communication using Multiple Antennas," in Proceedings of Intemational Symposium on Personal, Indoor and Mobile Radio Communications, vol. 1, (Hilton London Metropole Hotel, London, UK), pp. 698-703, IEEE, September 18-21 2000.

[4] P. Vandenameele, L. Van der Perre, M. Engels, B. Gyselinckx, and H. Man, "A novel class of uplink OFDM/SDMA algorithms: A statistical performance analysis," in Proceedings of Vehicular Technology Conference, vol. 1, (Amsterdam, Netherlands), pp. 324-328, IEEE, 19-22 September 1999.

[5] M. Münster and L. Hanzo, "Co-Channel Interference Cancellation Techniques for Antenna Array Assisted Multiuser OFDM Systems," in Proceedings of 3G - 2000 Conference, vol. 1, (London, Great Britain), pp. 256-260, IEE, March 27-29 2000.

[6] M. Sellathurai and S. Haykin, "A Simplified Diagonal BLAST Architecture with Iterative Parallel-Interference Cancellation Receivers," in Proceedings of International Conference on Communications, vol. 10, (Helsinki, Finnland), pp. 3067-3071, IEEE, June 11-14 2001

[7] P. Vandenameele, L. D. Perre, M. Engels, B. Gyselinckx, and H. Man, "A Combined OFDM/SDMA Approach," IEEE Journal on Selected Areas in Communications, vol. 18, pp. 23122321, November 2000.

[8] M. Münster and L. Hanzo, "Performance of SDMA Multiuser Detection Techniques for Walsh-Hadamard-Spread OFDM Schemes," in Proceedings of IEEE VTC '01 Fall, vol. 4, (Atlantic City, USA), pp. 2319-2323, IEEE, October 7-11 2001.

[9] L. Hanzo, T. Liew, and B. Yeap, Turbo Coding, Turbo Equalisation and Space-Time Coding for Transmission Over Fading Channels. New York, USA: IEEE Press - John Wiley \& Sons Ltd., 2002.

[10] G. Ungerböck, "Channel coding with multilevel/phase signals," IEEE Transactions on Information Theory, vol. IT-28, pp. 5567, January 1982.

[11] P. Robertson and T. Wörz, "Bandwidth efficient turbo trelliscoded modulation using punctured component codes," IEEE Journal on Selected Area on Communications, vol. 16, pp. 206218, February 1998.

[12] E. Zehavi, "8-PSK trellis codes for a Rayleigh fading channel," IEEE Transactions on Communications, vol. 40, pp. 873-883, May 1992.

[13] X. Li and J. Ritcey, "Bit-interleaved coded modulation with iterative decoding using soft feedback," IEE Electronics Letters, vol. 34, pp. 942-943, May 1998.

[14] “COST207, Digital Land Mobile Radio Communications," final report, Commission of the European Communities, Luxembourg, 1989. 